\title{
LA FORMATION DES ENSEIGNANTS GRECS DANS LA NOUVELLE ÉCOLOGIE ÉDUCATIVE
}

\author{
Vassilios Dagdilelis $^{1}$, Sophie Aslanidou ${ }^{2}$
}

\begin{abstract}
It is difficult, for the different actors of the Greek educational scene, to find agreements on the way to surpass an educational crisis due to the numerous political changes that shook the country latter decades. Internet (new phenomenon in the educational domain) provokes some «turbulences » in the distribution of a certain power in the heart of the sector.

Teachers in particular, and educators in general, are not ready to assume the too numerous changes that the introduction of new technologies brought in Greece. The permanent « goes and comes » on politics about the teachers' formation - this text will realize it - do not allow the Education Holders to fully insure a still complex - even elsewhere - role.

Even if researches multiply themselves on a more specialized formation in alphabetizing and in image-education, the official politic continues to introduce new medias in schools without insuring in parallel the formation of people who will be the instructors of such a progress.
\end{abstract}

\section{Introduction}

"L'éducation grecque se trouve dans une crise ». Ce sont des paroles que l'on entend de plus en plus souvent de la part des responsables de l'éducation grecque ces dix dernières années. 
Cette crise semble être non-uniforme et non-spécifique: Les divers acteurs de l'enseignement - enseignants, responsables politiques, administration, étudiants - la société même, ne semblent pas être en accord sur la crise elle-même et les moyens de la surpasser. Il y a cependant certains points communs dans cette opposition - et ce que nous appelons nouvelle écologie éducative constitue l'un de ces points.

Nous avons choisi d'utiliser le terme écologie pour souligner une caractéristique du système éducatif qui nous semble importante et nécessaire pour l'analyse d'une politique éducative. L'éducation constitue un système en soi, une espèce d'organisme complexe : elle est constituée de plusieurs parties, qui ont un but commun mais qui localement peuvent être antagonistes. L'introduction desdites nouvelles «technologies», pour donner un exemple, provoque des turbulences a l'intérieur du système éducatif puisque les nouvelles technologies, entre autres, changent la distribution des ressources disponibles et par conséquent transforment la distribution d'un certain pouvoir à l'intérieur du système. D'autre part, cette même éducation constitue un système qui interagit avec un autre, plus large, dans lequel l'éducation vit : la société elle-même. L'éducation doit donc être intégrée dans la société et prendre en compte les contraintes et les exigences de celleci : même si les Universités, pour donner un autre exemple, choisissent elles-mêmes leur propre enseignement, ces derniers temps on observe une très forte pression de la part de la société, pour que les Universités prennent en compte, dans leur planification, le «marché», c'est à dire le facteur économique. Nous voyons là une forte analogie avec les organismes vivants, dont l'étude se fait en prenant en compte à la fois leur «mécanisme» intérieur, mais leur environnement également.

Dans les paragraphes qui suivent nous allons présenter quelques éléments clés de cette nouvelle écologie. Cette dernière impose des nouvelles contraintes au système éducatif et nous pensons que la politique éducative grecque, telle qu 'elle se présente actuellement, n'est pas complètement adaptée aux circonstances et ne peut donc les surpasser. Pour illustrer notre position, nous proposerons une étude exemplative de la formation des enseignants grecs. 


\section{Quelques éléments clefs de la nouvelle écologie}

Il y a au moins deux éléments importants, nouveaux, qui influencent l'éducation à plusieurs niveaux: les nouvelles technologies et l'Union Européenne (UE).

Les nouvelles technologies constituent déjà un facteur presque révolutionnaire. La complexité didactique de leur utilisation en classe est très considérable: avec l'utilisation des nouvelles technologies - l'informatique surtout - l'organisation d'un cours devient une tâche plus compliquée que dans un cours «classique». L'enseignant doit organiser la situation en classe en intégrant un élément très sophistiqué, techniquement, dont la valeur didactique $\mathrm{n}$ 'est pas assurée par sa seule présence : pour le dire autrement, l'utilisation des nouvelles technologies ne garantit pas automatiquement le succès du cours. Il est évident que ces nouvelles technologies imposent une formation supplémentaire pour les enseignants.

La problématique de l'alphabétisation aux nouvelles technologies, y compris aux médias, est nouvelle en Grèce, mais elle commence à être impérative étant donnée l'entrée massive de l'informatique dans l'éducation. Les initiatives sont personnelles : il n'existe pas de politique de formation issue du Ministère de l'Education. Au plan officiel, ces questions restent plutôt inconnues.

Pourtant on peut citer quelques initiatives qui assurent l'implantation d'une éducation aux médias et aux nouvelles technologies.

Ainsi «The European Children Television Center (ECTC), organisme privé, travaille avec les enfants auprès des différentes municipalités sur l'Education aux Médias :

Le programme MELINA est un programme Européen dépendant du ministère de la culture, et travaille auprès des écoles primaires sur l'alphabétisation à l'image. C'est un programme qui vise à initier les enfants au monde des nouvelles technologies.

On peut ajouter à ceux-ci le programme du ministère de la Culture, «Allons au cinéma », une initiative du Festival International du cinéma de Thessaloniki qui s adresse aux jeunes des 9 à 17 ans et qui vise à éduquer le « regard » des jeunes et à leur apprendre à lire les images cinématographiques.

Dans l'enseignement supérieur, deux cours dans l'ensemble du pays assurent l'éducation aux médias : un au département de l'âge prés- 
colaire de l'Université de Crète, le second à ASPETE (Ecole Supérieure de Formation des Maîtres) à Thessaloniki.

L'intérêt d'une formation des enseignants à une éducation aux nouvelles technologies est impératif, et les problèmes que les enseignants rencontrent, dûs au manque d'une telle formation, s accumulent : Les raisons de la nécessité d'un programme plus global et d'une initiative officielle se multiplient également

En ce qui concerne l'UE, on est face à des phénomènes de nature différente, mais qui exigent également un changement radical de mentalité de la part des enseignants - de l'administration aussi. L'UE intervient souvent de façon indirecte - mais décisive - dans les systèmes éducatifs nationaux en traçant les buts de l'éducation pour les années à venir. Pour ne donner qu'un exemple, le changement de statut des Institutions Techniques Supérieures en Grèce (TEI), qui sont reconnues depuis trois ans comme équivalentes aux Universités... Mais sans l'accord, pour être plus précis avec le désaccord absolu, de la plupart des Universités. A un autre niveau, on peut aussi citer le fait que l'UE donne aux écoles la possibilité de financer leur fonctionnement de façon directe ou indirecte (à travers divers programmes). Or cette possibilité nécessite une recherche de ces possibilités - donc les enseignants et l'administration éducative doivent connaître la façon et l'endroit de ces financements potentiels.

Dans ce cadre, il est clair que le système éducatif doit, face à cette nouvelle écologie, préparer les futurs enseignants, les former, ainsi que l'administration actuellement en place.

\section{La formation des enseignants grecs}

En Grèce, le problème de la formation des enseignants et en particulier de leur formation pédagogique qui est en rapport avec les fonctions sociales, constitue une de présuppositions pour la réforme de l'école. Les provocations internationales, les évolutions sociales en général, et le développement de Sciences de l'Education reposent la question de la formation des enseignants d'une manière décisive qui, à cause de sa 
complexité, prend des dimensions de plus en plus larges. Les questions que la Science de l'éducation pose en Grèce peuvent se résumer ainsi :

- Quelles matières pourrait comprendre la formation des enseignants?

- Quel devrait être le profil d'un enseignant - quel enseignant a besoin de l'Education d'aujourd'hui ?

Une telle planification pourrait comprendre

- La connaissance de la matière enseignée. Actuellement, ce savoir repose uniquement sur les études de bases universitaires.

- La connaissance de l'école, c'est-à-dire un savoir qui repose sur le système d'éducation en rapport avec son rôle sociopolitique et culturel.

- La connaissance sur la didactique. Ce savoir est en rapport avec leur formation pédagogique dans le domaine de la didactique : psychologie, sociologie de l'éducation, évaluation.

Souvent la formation des enseignants a été le champ d'une confrontation idéologique. Les changements qui ont été proposés jusqu'à maintenant étaient fragmentaires, occasionnels, et ont été mis en place dans le cadre de réformes générales de l'enseignement secondaire et primaire.

Ce retard est dû à des raisons historiques et politiques en relation avec le développement des Sciences de l'Education et la politique éducative des gouvernements d'après-guerre. Point important d'un changement de la politique de l'éducation : la réforme de 1976-77, qui a entamé un dialogue sur le rôle et la formation des enseignants. Il commençait ainsi une démarche de remise en question de l'image et du rôle de l'enseignant. Les organisations syndicales, les politiciens et les experts ont alors organisé des séminaires et congrès sur des sujets concernant la formation de base et la formation continue des enseignants.

L'opinion publique traditionnelle, jusqu'à cette période, mettait en valeur le prestige de l'enseignant et insistait sur son rapport social. La politique officielle ne considérait pas l'enseignant comme un professionnel mais insistait sur l'aspect moral et éthique de la profession. Dans cette optique, il était fréquent de désigner les enseignants comme « des piliers de la nation » ou comme « des modèles moraux ». Souvent, les textes pédagogiques destinés aux enseignants sont connotés moralistes. 
Après 1976, commence une discussion sur une éducation plus élaborée et plus scientifique des enseignants.

Mais il faut distinguer les enseignants du primaire et ceux du secondaire. La formation des enseignants du primaire, jusqu'à 1982, avait eu lieu dans les écoles Normales, créées en Grèce en 1933. La formation durait alors deux ans. En 1964, cette formation dure trois ans ; la dictature de 1967 la remet à deux ans. Depuis 1982, les écoles Normales entrent dans l'enseignement supérieur et deviennent départements pédagogiques du primaire et de l'âge préscolaire. Depuis lors, tous les enseignants du primaire ont une formation identique, qui dure 4 ans, et un titre qui a la même valeur. Cette transformation a été interprétée comme une stratégie qui avait pour objectif de former des enseignants qui pourraient prendre le contrôle de leur travail, plus autonomes et plus conscients.

Dans l'enseignement secondaire, les enseignants des matières générales (langues, mathématiques, physique, chimie, etc.) suivent la formation initiale de quatre ans, dans leurs universités, mais leur formation pédagogique se différencie. Ce dernier point était toujours discuté auprès des organisations syndicales des enseignants mais aussi auprès des responsables politiques. Ces enseignants ont subi un ensemble de réformes qui essayaient de répondre au problème de l'absence de formation pédagogique.

En 1979, la création de SELME organise la formation pédagogique des enseignants pour une année scolaire. La participation était volontaire et toute organisation dépendait du Ministère de l'éducation Nationale La structure était très rigide mais était modifiée chaque fois qu'il avait un changement au Ministère de l'Education Nationale. Le choix des enseignants se faisait par tirage au sort: un grand nombre d'enseignants étaient exclus de cette formation. Quand elle a été transformée en 1992, seulement $2 \%$ des enseignants avaient été formés. A cette date, SELME se transforme en PEK, formation rapide de trois mois au départ, plus tard réduite à deux et actuellement d'une semaine seulement!

Aujourd'hui encore, le problème de la formation pédagogique continue à être discuté. Les propositions se succèdent, mais la discontinuité du système politique n'assure pas une position stable et une plus grande élaboration de la formation pédagogique. Mais le sujet même de la formation pose une difficulté énorme, parce qu'il faut prendre en considération le grand éventail des matières enseignées dans le secon- 
daire, qui comprend des théologiens, des mathématiciens, des professeurs de langues, etc.

Dans l'enseignement technique et professionnel, la formation pédagogique a eu une plus grande continuité. Ces enseignants (qui ont une maîtrise d'ingénieur, de médecine, d'économie, de sciences politiques ou de sociologie) reçoivent une formation pédagogique d'une année, dans une école supérieure de formation intitulée ASPETE (ex SELETE). La formation, obligatoire, comprend toutes les disciplines enseignées dans un département de science de l'éducation (sociologie ; méthodologie de la recherche, technologie de l'éducation, didactique, informatique, philosophie et management de l'éducation). Pourtant, bien que le problème de la formation pédagogique semble alors résolu, il reste un travail important en ce qui concerne la dimension pédagogique du métier d'enseignant Il est en effet très difficile à un ingénieur ou à un juriste de comprendre l'importance pédagogique que prend dans un champ anthropologique le métier d'enseignant, tout simplement parce qu'ils ne considèrent pas cet emploi comme une vocation...

D'une manière générale, la formation des enseignants a pour objectif de soutenir leur formation initiale.

\section{Quelques éléments de synthèse}

La formation des enseignants grecs constitue un exemple flagrant de l'inadaptation du système éducatif grec a la nouvelle écologie éducative. Son histoire de ces 20 dernières années montre clairement les raisons pour lesquelles le système ne peut pas s'adapter : une très forte dépendance à une certaine idéologie, une dépendance non moins forte aux changements politiques (plus précisément aux nombreux changements d'acteurs politiques de haut niveau : gouvernement, Premier ministre, ministre de l'Education nationale), absence d'une stratégie, d'une ligne directrice pour les années à venir.

Notre exemple dans cet article a été la formation des enseignants, mais d'une façon générale, les facteurs déterminants de cette formation mentionnés ci-dessus définissent de façon implicite le cadre général dans lequel s'inscrit le système éducatif grec. Ces facteurs, sans nul doute, interviennent dans le fonctionnement du système éducatif dans la plupart des pays - probablement tous. Cependant, ce qui est caractéristique pour la Grèce, est que ces facteurs s'imposent avec une force 
si grande qu'elle presque anéanti l'influence des autres composantes du système - tel le discours scientifique, ou les besoins futurs de l'économie grecque.

Dans ce cadre, il nous semble, qu'un changement radical du système éducatif grec s'impose comme absolument nécessaire.

\section{Notes}

${ }^{1}$ Université de Macédoine, Thessaloniki, Grèce

2 ASPETE, Ecole supérieure de formation des maîtres, Thessaloniki, Grèce 